Sādhanā Vol. 38, Part 4, August 2013, pp. 645-651. (C) Indian Academy of Sciences

\title{
A new universal gate for low power SoC applications
}

\author{
M GEETHA PRIYA $^{1, *}$ and K BASKARAN ${ }^{2}$ \\ ${ }^{1}$ Department of Electronics and Communication Engineering, Amrita Vishwa \\ Vidyapeetham, Coimbatore 641 112, India \\ ${ }^{2}$ Department of Computer Science and Engineering, Government College \\ of Technology, Coimbatore 641013 , India \\ e-mail: geetha.sri82@gmail.com; baski_101@gmail.com
}

MS received 3 September 2012; revised 2 December 2012; accepted 23 April 2013

\begin{abstract}
This paper formulates a new design technique for an area and energy efficient Universal NAND gate. The proposed robust three transistors (3T) based NAND gate is just as effective for dynamic power control in CMOS VLSI circuits for System on Chip (SoC) applications. The 3T NAND gate is intuitively momentous and lead to better performance measures in terms of dynamic power, reduced area and high speed while maintaining comparable performance than the other available NAND gate logic structures. Simulation tests were performed by employing standard Berkeley Predictive Technology Model (BPTM) $22 \mathrm{~nm}, 45 \mathrm{~nm}$ and $90 \mathrm{~nm}$ process technologies. The experiment and simulation results show that, the proposed 3T NAND gate effectively outperforms the basic CMOS NAND gate with excellent driving capability and signal integrity with exact output logic levels.
\end{abstract}

Keywords. Low power; CMOS; pass-transistor; NAND gate; Koomey's law.

\section{Introduction}

Not so long ago, researches were focused on developing portable applications in pursuit of high performance, thus increasing system complexity. Natural fallout of this process has been abridged battery life and long term reliability issues. Analysis of such systems, points to increased power consumption as the main factor (Rabaey \& Pedram 1996). Reduction in feature size also causes tunnelling effects. These realizations spurred interest in the area of low power design consequently creating a new field of electronics. Therefore along with area and speed, power consumption is an important criterion in measuring system performance (Goel et al 2006; Mariano \& Monico 2011; Baskaran et al 2011).

The significance of a universal gate is that programmable chips can be made of only one kind of gate, either NAND or NOR gate making manufacturing process simple and reducing the number of different processors required to make a computer. The benefit of utilizing a combination

*For correspondence 
of low-power components in conjunction with low-power design methodologies is more important now than ever before. Low power requirements continue to grow extensively as components become battery-powered, smaller and require more functionality. Koomey's law describes a long-term trend in the history of computing hardware and has actually been somewhat faster than Moore's law. Jonathan Koomey articulated the trend as follows: 'at a fixed computing load, the amount of battery you need will fall by a factor of two every year and a half' (Koomey Jonathan et al 2011).

In order to realize ultra low power SoC applications, circuits should be operated with low power dissipating gates. In reality, digital control systems have been designed approximately with either NAND or NOR gates, all the essential logic functions being derived from collections of interconnected NANDs or NORs. Low power and high reliability needs along with cost/performance advantages make NAND flash memory the ideal data storage solution for portable electronic devices. Designers are progressively more turning to embedded NAND solutions for dynamic, on-board storage. To support the ever-changing needs of current and emerging applications, the authors have developed the most appropriate NAND solution for these applications.

In this work, we present a circuit level design technique for universal NAND gate that reduces the overall dynamic power with reduced switching transistor count. The proposed design of 3T NAND gate is based on CMOS inverter and Pass Transistor Logic. The rest of the paper is organized as follows, in section 2, a review of CMOS Inverter and Pass Transistor Logic (PTL) is presented. In section 3, the proposed work on a 3T NAND gate is presented, which is followed by the simulation results and conclusions in sections 4 and 5 , respectively.

\section{Review of CMOS inverter and PTL}

CMOS inverters are some of the most largely used and adaptable MOSFET inverters in chip designing. They operate with minimal power loss and at relatively high speed (Franco Maloberti 2001). Furthermore, the CMOS inverter has good logic buffer characteristics in that. Its noise margins are large in both low and high states. A CMOS inverter shown in figure 1 contains a PMOS and a NMOS devices connected at the drain and gate terminals, a supply voltage VDD



Figure 1. CMOS inverter. 


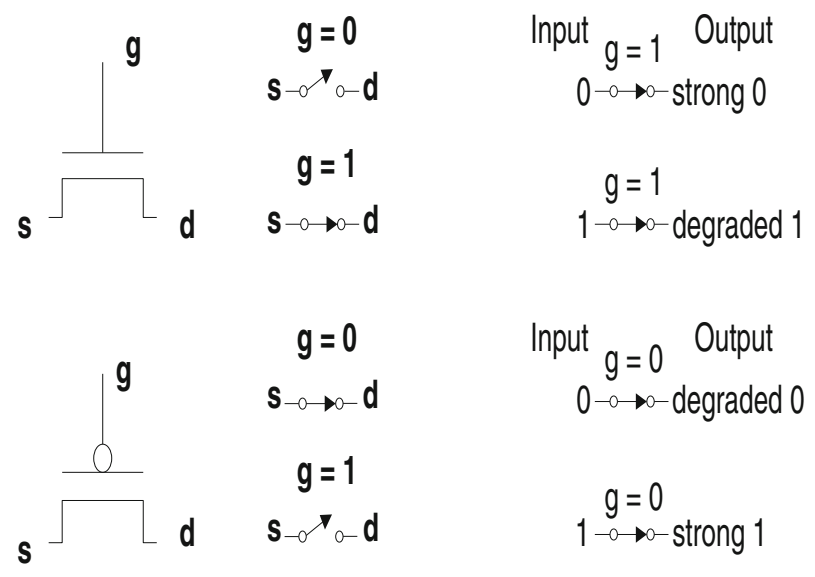

Figure 2. Pass transistor logic.

at the PMOS source terminal, and a ground connected at the NMOS source terminal, were IN is connected to the gate terminals and OUT is connected to the drain terminals (Franco Maloberti 2001). As the input to the inverter varies between logic 0 and logic 1 , the state of the NMOS and PMOS varies accordingly to make output as logic 1 and logic 0 , respectively.

The most popular and widely used alternative to complementary CMOS is pass-transistor logic. Pass-transistor logic attempts to decrease the quantity of transistors necessary to realize the logic by allowing the principal inputs to drive gate terminals (Radhakrishnan et al 1985; Srinivasan et al 2012) as well as drain/source terminals as shown in figure 2. It is observed from figure 2 that when the device getting used as a pass-transistor may conduct current in either direction. The NMOS transistors pass a strong logic 0 but a weak logic 1 (threshold voltage drop. High $=$ VDD $-\mathrm{V}_{t h n}$ ) and PMOS transistors pass a strong logic 1 but a weak logic 0 (threshold voltage drop. Low $=\mathrm{V}_{t h p}$ ). Thus, NMOS switches are best for pull-down network and the PMOS switches are best for pull-up network.

\section{Proposed 3T NAND design}

The new design of 3T NAND gate is shown in figure 3. The high density layout, speed and compact design advantages of PTL and CMOS inverter design style can be utilized efficiently to design 3T NAND circuit. The 3T NAND functionality can be explained as follows.

- The PMOS M1 and NMOS M2 on the left form a modified CMOS inverter structure. The PMOS M3 on the right acts as a pass transistor.

- When $A=1, M 3$ is OFF and the modified inverter on the left (M1 \& M2) functions as a normal CMOS inverter. Therefore, the output is the complement of input B.

- When $\mathrm{A}=0$ \& $\mathrm{B}=0, \mathrm{M} 2$ is $\mathrm{OFF}, \mathrm{M} 1$ and $\mathrm{M} 3$ are $\mathrm{ON}$ which leads to an undefined output state ' $\mathrm{X}$ ', because M1 tends to pull down the output node while M3 tends to pull up the output node.

Similarly when $\mathrm{A}=0 \& \mathrm{~B}=1, \mathrm{M} 1$ is $\mathrm{OFF}, \mathrm{M} 2$ and $\mathrm{M} 3$ are $\mathrm{ON}$ leading to an undefined output state ' $\mathrm{X}$ ', because M2 tends to pull down the output node while M3 tends to pull up the output node. 




Figure 3. $3 T$ NAND.



GND

Figure 4. AND gate.

- For $\mathrm{A}=0 \& \mathrm{~B}=0$ or 1 , a strong logic ' 1 ' output is required. It is possible to obtain exact output logic levels with the proposed circuit, if the channel width of M3 is made 6 times that of M2 or 3 times that of M1. That is

$$
\mathrm{W}_{\mathrm{M} 3}=6 \times \mathrm{W}_{\mathrm{M} 2}=3 \times \mathrm{W}_{\mathrm{M} 1} .
$$

Thus, M3 becomes much stronger than M1 and M2, giving a strong logic ' 1 ' at output when input $\mathrm{A}=0$.

The proposed 3T NAND gate is exempted from body bias effect, as there is no stacking of transistors. Exact output logic levels are attained for all the input combinations without any voltage degradation. An AND gate operation could be obtained with an additional CMOS inverter at the NAND output with 5 transistors totally as shown in figure 4.

\section{Simulation results}

The Performance of the 3T NAND gate is evaluated based on its power dissipation, delay and power delay product (PDP) compared with conventional CMOS four transistors (4T) NAND gate. Schematics are designed for all circuits using Custom Designer in Synopsys for TSMC $0.18 \mu \mathrm{m}$ technology. The original netlists obtained from the schematics are modified according to BPTM $22 \mathrm{~nm}$ process technology (Geetha Priya et al 2012). The modified netlists are simulated using Synopsys HSPICE for power and delay estimations. 
Table 1. Simulation results of NAND gates compared (power in $\mathrm{W}$, delay in $\mathrm{pS}$ and PDP in J).

\begin{tabular}{|c|c|c|c|c|c|c|}
\hline \multirow{3}{*}{$\begin{array}{l}\text { Process Tech. } \\
\text { Parameters }\end{array}$} & \multicolumn{2}{|c|}{$90 \mathrm{~nm} @ \mathrm{VDD}=1.2 \mathrm{~V}$} & \multicolumn{2}{|c|}{$45 \mathrm{~nm} @ \mathrm{VDD}=0.5 \mathrm{~V}$} & \multicolumn{2}{|c|}{$22 \mathrm{~nm} @ \mathrm{VDD}=0.2 \mathrm{~V}$} \\
\hline & & 4T CMOS & & 4TCMOS & & 4TCMOS \\
\hline & 3T NAND & NAND & 3T NAND & NAND & 3T NAND & NAND \\
\hline Dynamic power & 8.4200 E-09 & 10.2812 E-09 & $4.9058 \mathrm{E}-11$ & $6.1356 \mathrm{E}-11$ & $1.8661 \mathrm{E}-12$ & $2.4743 \mathrm{E}-12$ \\
\hline Delay & 54.8 & 75.2 & 43.6 & 59.4 & 38.2 & 47.1 \\
\hline $\mathrm{PDP}_{\text {dynamic }}$ & 461.4 E-21 & $773.1 \mathrm{E}-21$ & $213.8 \mathrm{E}-23$ & $364.4 \mathrm{E}-23$ & $71.2 \mathrm{E}-24$ & 116.5 E-24 \\
\hline
\end{tabular}

All devices used are of standard threshold voltage (Vth) and the operating temperature taken is $27^{\circ} \mathrm{C}$. The $3 \mathrm{~T}$ NAND gate ensures optimum performance for frequency range between $50 \mathrm{MHz}$ and $0.3 \mathrm{GHz}$. All the results were simulated and analysed in the range of $125 \mathrm{MHz}$ to $0.25 \mathrm{GHz}$. Table 1 shows the simulation results of 3T \& 4T NAND gates compared at $90 \mathrm{~nm}, 45 \mathrm{~nm}$ and $22 \mathrm{~nm}$ process technology (Zhao \& Cao 2006). Dynamic power dissipation is estimated by applying all four possible input vectors $(00,01,10$ and 11) arbitrarily. Table 2 gives the simulation results of $3 \mathrm{~T}$ NAND at $22 \mathrm{~nm}$ process for various supply voltages $(0.2 \mathrm{~V}, 0.45 \mathrm{~V}, 0.6 \mathrm{~V}$ and $0.75 \mathrm{~V})$.

For $0.022 \mu \mathrm{m}(22 \mathrm{~nm})$ process, the length of the channel for all transistors was taken as $\mathrm{L}=$ $0.022 \mu \mathrm{m}$, channel width of $\mathrm{M} 1, \mathrm{~W}_{\mathrm{M} 1}=0.200 \mu \mathrm{m}$, channel width of $\mathrm{M} 2, \mathrm{~W}_{\mathrm{M} 2}=0.100 \mu \mathrm{m}$, and channel width of $\mathrm{M} 3, \mathrm{~W}_{\mathrm{M} 3}=0.600 \mu \mathrm{m}$.

For $0.045 \mu \mathrm{m}(45 \mathrm{~nm})$ process, the length of the channel for all transistors was taken as $\mathrm{L}=$ $0.045 \mu \mathrm{m}$, channel width of $\mathrm{M} 1, \mathrm{~W}_{\mathrm{M} 1}=1.000 \mu \mathrm{m}$, channel width of $\mathrm{M} 2, \mathrm{~W}_{\mathrm{M} 2}=0.500 \mu \mathrm{m}$, and channel width of $\mathrm{M} 3, \mathrm{~W}_{\mathrm{M} 3}=3.000 \mu \mathrm{m}$.

For $0.090 \mu \mathrm{m}(90 \mathrm{~nm})$ process, the length of the channel for all transistors was taken as $\mathrm{L}=$ $0.090 \mu \mathrm{m}$, channel width of $\mathrm{M} 1, \mathrm{~W}_{\mathrm{M} 1}=2.000 \mu \mathrm{m}$, channel width of $\mathrm{M} 2, \mathrm{~W}_{\mathrm{M} 2}=1.000 \mu \mathrm{m}$, and channel width of $\mathrm{M} 3, \mathrm{~W}_{\mathrm{M} 3}=6.000 \mu \mathrm{m}$.

Figure 5 shows transfer characteristics for proposed 3T NAND gate with perfect output logic levels without any voltage degradation. Figure 6 gives the power savings of 3T NAND gate showing an improvement of more than $22 \%$ of less power consumption over conventional CMOS NAND Gate (4T) as the process technology diminishes.

From table 1, we can state the following.

- Considering the power dissipation, 3T NAND gate shows less dynamic power dissipation compared to conventional CMOS NAND gate. The reduction in dynamic power dissipation comes from the fact of reduced internal capacitances as transistors count is less, which results in less glitches at the outputs.

Table 2. Simulation results of 3T NAND gate @ $0.022 \mu \mathrm{m}(22 \mathrm{~nm})$ for various supply voltages (supply voltage in $\mathrm{V}$, power in $\mathrm{W}$ and delay in $\mathrm{pS}$ ).

\begin{tabular}{lcccccc}
\hline \multirow{2}{*}{ Supply voltage } & Dynamic power & Delay & \multicolumn{4}{c}{ Average power for various input vectors } \\
\cline { 4 - 7 } & & & 00 & 01 & 10 & 11 \\
\hline 0.2 & $1.0661 \mathrm{E}-12$ & 38.2 & $3.0021 \mathrm{E}-13$ & $5.8750 \mathrm{E}-13$ & $1.7239 \mathrm{E}-13$ & $1.4215 \mathrm{E}-14$ \\
0.45 & $5.8167 \mathrm{E}-12$ & 37.4 & $6.7812 \mathrm{E}-13$ & $9.0081 \mathrm{E}-13$ & $5.0414 \mathrm{E}-13$ & $4.6900 \mathrm{E}-14$ \\
0.6 & $10.6535 \mathrm{E}-12$ & 37.0 & $9.3406 \mathrm{E}-13$ & $11.1775 \mathrm{E}-13$ & $8.6580 \mathrm{E}-13$ & $7.8640 \mathrm{E}-14$ \\
0.75 & $16.3971 \mathrm{E}-12$ & 36.6 & $11.3810 \mathrm{E}-13$ & $12.3462 \mathrm{E}-13$ & $9.4174 \mathrm{E}-13$ & $10.8979 \mathrm{E}-14$ \\
\hline
\end{tabular}




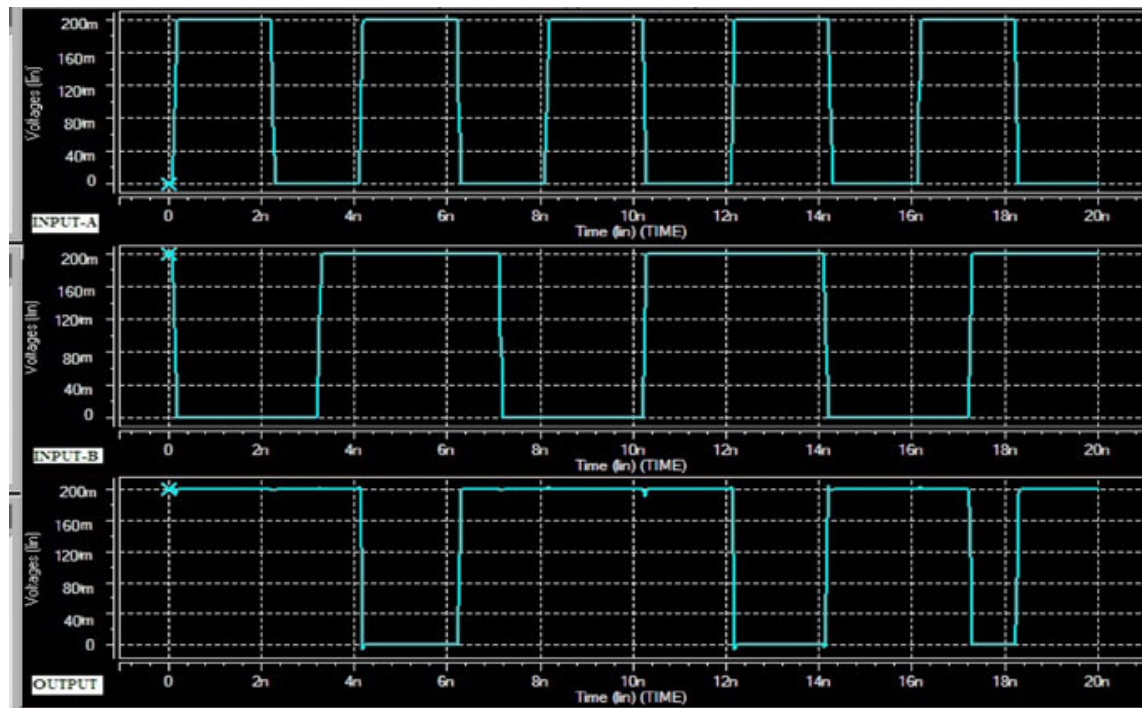

Figure 5. Transfer characteristics of 3T NAND gate @ $22 \mathrm{~nm}$.

- With regards to the speed, it can be seen that the benefit of the pass transistor logic structure introduced here, exhibiting the smallest propagation delay than CMOS NAND gate.

- The power-delay product (PDP) column confirms the energy-efficiency for the 3T NAND gate built using the new logic structure. This presents the lowest PDP metric, due to the combined reduction of power consumption and propagation delay.

From table 2, we can state the following.

- We have carried out a separate set of simulations to determine the maximum power supply voltage that 3T NAND gate can tolerate at $0.022 \mu \mathrm{m}(22 \mathrm{~nm})$ process technology while maintaining its correct functionality. As shown in column supply voltage, the proposed NAND can operate properly with 0.2 to $0.8 \mathrm{~V}$ supply voltage.

\section{Power Savings}



Figure 6. Power savings of 3T NAND gate. 
- With increase in supply voltage, the dynamic power increases and the delay improves.

- The average power column for different input vectors shows the fact that subthreshold leakage is dependent on input vectors.

- With respect to the implementation area, it can be seen that the proposed NAND gate required the smallest area due to less transistor count, which can also be considered as one of the factors for presenting lower delay and power consumption.

- It is a good candidate for battery-operated applications where low dissipation modules with standby modes are required.

\section{Conclusion}

This paper presents features of 3T NAND gate based on PTL and CMOS logic. HSPICE simulations at $22 \mathrm{~nm}$ showed power savings up to $32.6 \%$ and speed improvements up to $23 \%$, for a joint optimization of $63 \%$ for the PDP. Throughout logic design, the proposed 3T NAND gate could be used to reduce the dynamic power of VLSI SoC circuits.

\section{References}

Baskaran K, Geetha Priya M and Krishnaveni D 2011 Leakage power reduction techniques in deep submicron technologies for VLSI applications. Proceedings of International Conference on Communication Technology and System Design, Elsevier- Procedia Engineering, 30:1163-1170

Franco Maloberti 2001 Analog design for CMOS VLSI systems. The Netherlands: Kluwer Academic Publishers

Geetha Priya M, Baskaran K, Krishnaveni D and Srinivasan S 2012 A new leakage power reduction technique for CMOS VLSI circuits. Journal of Artificial Intelligence, 5: 227-232

Goel Sumeer, Ashok Kumar and Magdy A Bayoumi 2006 Design of robust, energy-efficient full adders for deep-submicrometer design using hybrid-CMOS logic style. IEEE Transactions on VLSI Systems, 14: $1309-1321$

Koomey Jonathan, Berard Stephen, Sanchez Marla and Wong Henry 2011 Implications of historical trends in the electrical efficiency of computing. Annals of the History of Computing, IEEE, 33: 46-54

Mariano Aguirre-Hernandez and Monico Linares-Aranda 2011 CMOS full-adders for energy-efficient arithmetic applications. IEEE Transactions on Very Large Scale Integration Systems, 19:718-721

Rabaey Jan M and Pedram Massoud 1996 Low power design methodologies. Boston: Kluwer Academic Publishers

Radhakrishnan D, Whitaker SR and Maki GK 1985 Formal design procedures for pass transistor switching circuits. EE J. Solid State Circuits, 20:531-535

Srinivasan S, Geetha Priya M and Baskaran K 2012 PTL/CMOS Logic based full adder for high speed applications. International Journal of Advanced Computing, 35:84-87

Zhao W and Cao Y 2006 New generation of predictive technology model for sub-45 nm early design exploration. IEEE Transactions on Electron Devices, 53: 2816-2823 